\title{
A Japanese man with malaria tests negative for fever after spending 6 months in rural Kenya
}

\author{
Takashi Sugimoto ${ }^{1}$, Kensuke Takahashi ${ }^{1,2}$, Kosuke Matsui ${ }^{1}$, Masahito Asada ${ }^{3}$, Osamu Kaneko ${ }^{3}$ and \\ Koya Ariyoshi, ${ }^{1,2^{*}}$ (D)
}

\begin{abstract}
A previously healthy Japanese man in his fifties was admitted to our hospital because of a recurrent fever after returning from Kenya and Madagascar. He was ambulant with a body temperature of $36.6^{\circ} \mathrm{C}$. His physical examination revealed normal except for tender hepatomegaly. The blood test results showed no apparent abnormality except thrombocytopenia and mild liver dysfunction. The rapid diagnostic test and Giemsa-stained blood film were repeatedly negative for malaria. Computed tomography scans of the chest, abdomen, and pelvis revealed no significantly abnormal findings.
\end{abstract}

\section{History}

A previously healthy Japanese man in his fifties was admitted to the Department of Infectious Disease at Nagasaki University Hospital in Japan because of a fever after returning from a 6-month stay in Kenya. The patient stayed in Madagascar from November 20YY-1 to January $20 \mathrm{YY}$ prior to his stay a rural city in Kenya from January to late June $20 \mathrm{YY}$ to conduct behavioral research at a community hospital. According to the patient, the hospital ward consisted of many febrile patients, including malaria patients, but his research work did not involve direct contact with any patients. He also reported having many mosquito bites and walking on muddy roads since it was the rainy season. He drank tea with unpasteurized milk from water buffalo for breakfast, ate local food for lunch, and cooked supper for himself. He drank bottled water throughout the stay. He was vaccinated against hepatitis A and $\mathrm{B}$, tetanus, rabies, typhoid fever, and yellow fever prior

\footnotetext{
* Correspondence: kari@nagasaki-u.ac.jp

'Department of Infectious Diseases, Nagasaki University Hospital, Nagasak

University, Nagasaki, Japan

${ }^{2}$ Department of Clinical Medicine, Institute of Tropical Medicine, Nagasaki

University, Nagasaki, Japan

Full list of author information is available at the end of the article
}

to his travel, but he had not taken anti-malarial chemoprophylaxis. He denied having sexual contact.

Twenty days (day $\mathrm{x}-8$ ) after returning to Japan, he experienced episodes of fever with headache, fatigue, and back pain. Five days before admission, his body temperature increased to more than $38{ }^{\circ} \mathrm{C}$, and he had frequent urination. On the following day, he visited a local clinic where he was prescribed levofloxacin and acetaminophen and diagnosed with urinary tract infection. Two days before admission, his temperature increased to $39.6{ }^{\circ} \mathrm{C}$ at night and was accompanied by chills; thus, he visited our outpatient clinic on the next day (day $x-1)$.

\section{Clinical examination}

Upon examination at the first visit, he was ambulant with a body temperature of $36.6{ }^{\circ} \mathrm{C}$, a blood pressure of $108 / 85 \mathrm{mmHg}$, a pulse of $85 \mathrm{bpm}$, and a respiratory rate of 14 per minute. His abdominal examination revealed tender hepatomegaly. There was no rash, no lymphadenopathy, no bleeding tendency, and no joint pain. The remainder of the physical examination results was normal. The blood test results showed no apparent abnormality except moderate thrombocytopenia and mild liver dys-

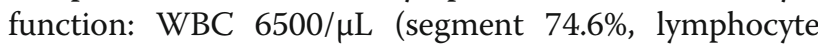


18.9\%), hemoglobin $14.0 \mathrm{~g} / \mathrm{dL}$, platelets $7.4 \times 10^{4} / \mu \mathrm{L}$, total bilirubin $0.8 \mathrm{mg} / \mathrm{dL}$, AST $42 \mathrm{U} / \mathrm{L}$, ALT $50 \mathrm{U} / \mathrm{L}$, BUN $13 \mathrm{mg} / \mathrm{dL}$, and creatinine $0.77 \mathrm{mg} / \mathrm{dL}$. The urinalysis showed proteinuria $( \pm)$, occult blood in the urine $(-)$, and $\mathrm{WBC}(-)$. The rapid diagnostic test $\left(\right.$ BinaxNOW $\left.{ }^{\circ}\right)$ and Giemsa-stained blood film were negative for malaria. Computed tomography (CT) scans of the chest, abdomen, and pelvis revealed no significantly abnormal findings.

On day $\mathrm{x}$, the patient was admitted for further investigation. Intravenous ceftriaxone $2 \mathrm{~g}$ per day was initiated after two sets of blood cultures were taken. On the second hospital day $($ day $x+1)$, the rapid diagnostic test and Giemsa-stained blood test were repeated, and the results were negative. However, his body temperature increased again at night. On the third hospital day, the blood culture results were negative. Ceftriaxone was discontinued, and oral minocycline $100 \mathrm{mg}$ every $12 \mathrm{~h}$ was started. On the 5th hospital day $($ day $\mathrm{x}+4)$, his body temperature increased again at night (Fig. 1). Additional diagnostic tests were ordered.

\section{Clinical questions}

1. What are the important differential diagnoses?

2. Which diagnostic tests should be performed?

\section{Diagnosis and clinical course}

We requested to conduct a Brucella agglutination reaction test at an external commercial laboratory; an IgM immunofluorescent assay (IFA) and PCR assays for rickettsia and Borrelia at the national reference laboratory, the National Institute of Infectious Diseases in Tokyo; and PCR assays for malaria at the Institute of Tropical Medicine at Nagasaki University.

On the 6th hospital day $($ day $x+5)$, it was reported that PCR amplification targeting malaria cytb was positive, and the sequence analysis showed that it matched with Plasmodium malariae. The Giemsa-stained films of the blood samples were re-examined by an experienced malariologist, and they revealed a low level of malaria parasites with the proportion of erythrocytes ranging from $<0.006$ to $0.018 \%$. Thus, the patient was diagnosed with quartan malaria and treated with artemether/lumefantrine for 3 days. On the 9th hospital day, defervescence was achieved, and the patient was discharged. The results of the Brucella agglutination reaction (B. abortus and $B$. canis) were negative. Anti-rickettsia antibodies (Orientia species and Rickettsia japonica) and PCR assays for rickettsia and Borrelia species were not performed eventually because malaria was diagnosed, and the fever did not respond to minocycline. One and a half years after discharge, the patient remained free from recrudescence.

\section{Discussion}

In evaluating febrile patients who travelled to malariaendemic countries, it is imperative to pay special attention to exclude falciparum malaria, as it can rapidly progress and cause death unless the patient is promptly treated with anti-malaria drugs. Therefore, if there is any clinical suspicion of malaria, malaria tests

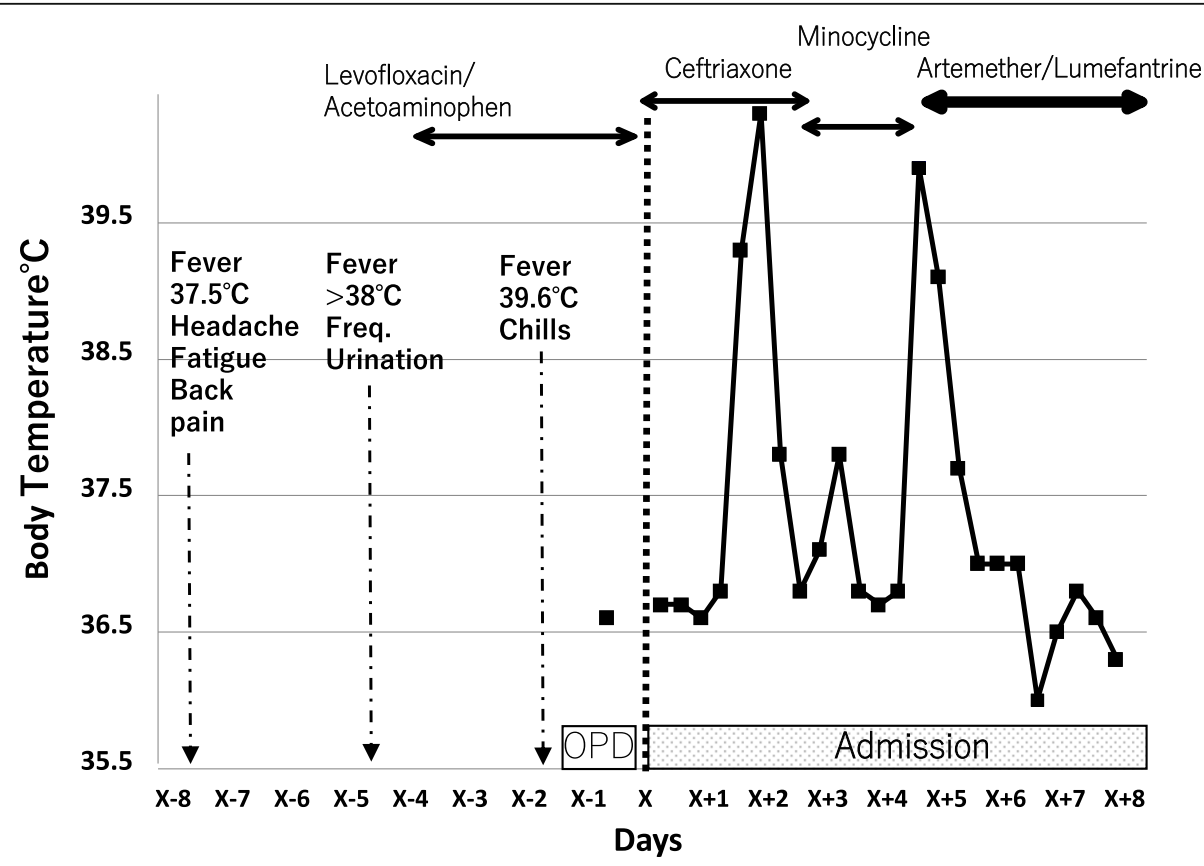

Fig. 1 Clinical course and body temperature 
should be repeated at least three times with 12- to 24$\mathrm{h}$ interval to exclude malaria [1].

In the current case study, malaria was the number one suspected disease among the probable differentials, and malaria tests, including the rapid diagnosis test and blood smear examination, were repeated and showed negative results. It is worth knowing that in the setting of travel medicine, sensitivity of rapid diagnosis tests for P. falciparum is significantly lower (median $74.1 \%$ ) if the parasitemia level was below $100 / \mu \mathrm{L}$ compared with above 100/ $\mu \mathrm{L}$ (median 94.3\%) [2] and that in cases of Plasmodium malariae infection, the parasitemia level remains very low; thus, the presence of parasitemia is often missed by malaria blood film tests $[3,4]$. Furthermore, the malaria rapid diagnosis test used in the current case is based on an immunochromatographic method with detection antibodies targeting both $P$. falciparum-specific histidine-richprotein-2 (PfHRP2) and a pan-malarial antigen, Plasmodium aldolase, but the sensitivity of this test is lower for non-falciparum malaria, especially in the detection of $P$. ovale and $P$. malariae, probably due to the lower affinity of some monoclonal antibodies to these species [5]. PCR assays, on the other hand, are capable of identifying malaria with both high sensitivity and high specificity. A disadvantage of PCR is that it is not rapid or a point of care in routine clinical practice. However, PCR needs to be conducted to diagnose conditions such as $P$. malariae infection, as the current case exemplifies. Would the patient have been treated empirically with antimalarial drugs if PCR assays had not been available? The answer highly depends on other factors, such as clinical severity, access to antimalarial drugs, and in this case, we would less likely have started empirical malaria treatment without knowledge of $P$. malariae disease.

He also had moderate thrombocytopenia, but we considered it rather non-specific reaction and did not investigate further, because his general condition was good, and DIC was excluded. It returned to normal rapidly following malaria treatment.

Treating a fever of an individual who has returned from traveling abroad, especially in tropical countries, is challenging for physicians working in high-income countries because (a) they have to diagnose tropical infectious diseases, for which clinicians often have little experience; (b) some tropical infectious diseases, such as falciparum malaria, are fatal; and (c) other diseases, such as typhoid fever, lead to significant public health threats to the local population. In approaching the fever of a returned traveller, thorough and directional evaluations are essential: comprehensive history taking, examinations to differentiate critical and curable diseases, and timely consultations with experts should be conducted when necessary. Collecting the latest epidemiological information of endemic diseases is also crucial. The prevalence of $P$. malariae is much lower than that of $P$. falciparum but not uncommon. According to recent reports from Kenya [6] and Madagascar [7], the prevalence of $P$. malariae among symptomatic patients was $3.3 \%$ and $1.2 \%$, respectively, while that of $P$. falciparum was $77 \%$ and $52 \%$, respectively. Moreover, the vast majority of $P$. malariae infections were coinfected with $P$. falciparum. Therefore, $P$. malariae infection is often overlooked and neglected in the local population.

Finally, the fever pattern should also be carefully observed. It was noted that the patient in this case study had a fever that was periodic with an exact 72-h interval, which could have been a clue for the diagnosis of quartan malaria (Fig. 1).

\section{Final diagnosis}

Quartan malaria (P. malariae infection)

\section{Abbreviations}

WBC: White blood cell; AST: Aspartate transaminase; ALT: Alanine transaminase; BUN: Blood urea nitrogen; CT: Computed tomography; IFA: Immunofluorescent assay; PCR: Polymerase chain reaction; PfHRP1: $P$. falciparum-specific histidine-rich-protein-2

\section{Acknowledgements \\ This is secondary publication. Much of the content in this manuscript was derived from a previously published case report in a Japanese commercial journal, IJISHINPOU. We thank the patient who agreed to publish his clinical information.}

\section{Authors' contributions}

TS and KA were involved in re-writing the original paper in English for this special issue. KT and KM were involved in the clinical management of the current case. KT and KA prepared the case report published originally in Japanese. MA and OK conducted malaria tests. The authors read and approved the final manuscript.

\section{Funding}

This manuscript did not require any funding.

\section{Availability of data and materials}

All data generated or analyzed during this study are included in this published article.

\section{Ethics approval and consent participate}

This case report does not require the Institutional Ethics Approval as this is a secondary publication, and a written informed consent was obtained from the patient before publication. All the co-authors approved the final draft of manuscript.

\section{Consent for publication}

Consent to publish was obtained from the IJISHINPOU, which published the original case report in Japanese.

\section{Competing interests}

The authors declare that they have no competing interests.

\section{Author details}

'Department of Infectious Diseases, Nagasaki University Hospital, Nagasaki University, Nagasaki, Japan. ${ }^{2}$ Department of Clinical Medicine, Institute of Tropical Medicine, Nagasaki University, Nagasaki, Japan. ${ }^{3}$ Department of Protozoology, Institute of Tropical Medicine, Nagasaki University, Nagasaki, Japan. 
Received: 2 April 2020 Accepted: 1 May 2020

Published online: 24 June 2020

\section{References}

1. Lalloo DG, Shingadia D, Bell DJ, et al. UK malaria treatment guidelines 2016. J Infect. 2016;72:635-49.

2. Maltha J, Gillet $P$, Jacobs J. Malaria rapid diagnostic tests in travel medicine. Clin Microbiol Infect. 2013;19:408-15.

3. Collins WE, Jeffery GM. Plasmodium malariae: parasite and disease. Clin Microbiol Rev. 2007;20(4):579-92.

4. Schindler T, Jongo S, Studer F, et al. Two cases of long-lasting, submicroscopic Plasmodium malariae infections in adults from coastal Tanzania. Malar J. 2019;18:149.

5. Grobusch MP, Hänscheid T, Zoller T, Jelinek T, Burchard GD. Rapid immunochromatographic malarial antigen detection unreliable for detecting Plasmodium malariae and Plasmodium ovale. Eur J Clin Microbiol Infect Dis. 2002;21(11):818-20.

6. Lo E, Nguyen K, Nguyen J, Hemming-Schroeder E, et al. Plasmodium malariae prevalence and csp gene diversity, Kenya, 2014 and 2015. Emerg Infect Dis. 2017;23(4):601-10.

7. Mehlotra RK, Howes RE, Cramer EY, et al. Plasmodium falciparum parasitemia and band sensitivity of the SD bioline malaria Ag P.f/Pan rapid diagnostic test in Madagascar. Am J Trop Med Hyg. 2019;100(5):1196-201.

\section{Publisher's Note}

Springer Nature remains neutral with regard to jurisdictional claims in published maps and institutional affiliations.

\section{Ready to submit your research? Choose BMC and benefit from:}

- fast, convenient online submission

- thorough peer review by experienced researchers in your field

- rapid publication on acceptance

- support for research data, including large and complex data types

- gold Open Access which fosters wider collaboration and increased citations

- maximum visibility for your research: over $100 \mathrm{M}$ website views per year

At BMC, research is always in progress.

Learn more biomedcentral.com/submissions 